\title{
Extending the dynamic range of microchannel plate detectors using charge-integration-based counting
}

\author{
Daniel J. Gershman ${ }^{1}$, Levon A. Avanov ${ }^{1,2}$, Dennis J. Chornay ${ }^{1,2}$, Amy C. Rager ${ }^{1,3}$, Craig \\ J. Pollock ${ }^{4}$, Guy Grubbs ${ }^{1,3}$, David A. Mackler ${ }^{1,3}$, Corey J. Tucker ${ }^{5}$, and Nikolaos P. \\ Paschalidis ${ }^{1}$ \\ ${ }^{1}$ NASA Goddard Space Flight Center, Greenbelt, MD, 20771, USA \\ ${ }^{2}$ Department of Astronomy, University of Maryland, College Park, MD, 20742, USA \\ ${ }^{3}$ Department of Physics, Catholic University of America, Washington DC, 20064, USA \\ ${ }^{4}$ Denali Scientific, Healy AK, 99743, USA \\ ${ }^{5}$ AS and D Inc., Greenbelt, MD, 20771, USA
}

\section{Corresponding author: Daniel J. Gershman (daniel.j.gershman@nasa.gov)}

\begin{abstract}
Microchannel plate (MCP) detectors provide a mechanism to produce a measureable current pulse ( $\sim 0.1 \mathrm{~mA}$ over several nanoseconds) when stimulated by a single incident particle or photon. Reductions of the device's amplification factor (i.e., gain) due to high incident particle flux can lead to significant degradation of detection system performance. Here we develop a parameterized model for the variation of MCP gain with incident flux. This model provides a framework with which to quantify the limits of high-flux MCP operation. We then compare the predictions of this model to laboratory measurements of an MCP's response to a pulsed charged particle beam. Finally, we demonstrate that through integration of the MCP output current in pulsed operation, effective count rates up to $\sim 1 \mathrm{GHz}$ can be achieved, more than an order of magnitude increase over conventional counting techniques used for spaceflight applications.
\end{abstract}

\section{Introduction}

Microchannel plate (MCP) detectors have become a standard technology for the measurement of individual photons or particles in space [1] MCPs consist of a regular array of cylindrical continuous dynode electron multipliers (CEMs) [2]. A single MCP plate can contain up to $10^{7}$ of these miniature CEMs, each with diameters of the order $\sim 10-100 \mu \mathrm{m}$ and lengths on the order of $\sim 1 \mathrm{~mm}$ [3]. When an electric potential of a few hundred volts is applied across the MCP plate, an incident particle or photon that strikes the inside of a channel can generate a secondary electron cascade. This cascade typically produces a cloud of only $\sim 10^{3}-10^{4}$ electrons on the output that is not readily distinguishable from electronic noise by charge-sensitive electronics. To increase the secondary electron yield per incident particle up to $\sim 10^{6}-10^{8} \mathrm{e}^{-}$, multiple MCP plates (typically 2 or 3 ) can be stacked in series, with multiple channels simultaneously excited in the bottom plates due to charge-cloud spreading [4,5]. The total amplification factor is commonly referred to as the 'gain,' and is crucial to characterize for a detection system. 
MCP gain is most strongly a function of the applied voltage, with further modulation from the initial secondary electron yield of the incident particle or photon and the depth within each channel of the bombardment $[3,6,7]$. Although there can be some variation in gain with incident particle properties [8-11], MCP detector systems can be configured to achieve a similar operational regime for both ions and electrons [7]. Each time an incident particle or photon initiates a cascade within a channel, charge is depleted from channel walls and must be replenished via the MCP's power supply. A simple model of an MCP describes each channel as a charge reservoir, with a characteristic charge replenishment $R C$ time scale, typically on the order of a few milliseconds, determined by the plate's resistance ( > $1 \mathrm{M} \Omega)$ and capacitance $(\sim 100 \mathrm{pF})$ [12]. In such a model, an incident particle entering a channel before it has fully recharged results in reduced MCP gain. However, because each channel acts somewhat independently, multiple incident particles can strike the MCP as long as each channel is depleted, on average, less than once per recharge time. The response of MCP gain to high incident particle flux has been studied in both steady-state [13-15] and impulsive [16-18] regimes. These investigations have demonstrated that the charge reservoir concept provides a reasonable representation of MCP behavior, and that maintaining a low ratio of incident particles-per-channel within a channel recovery time ensures limited detection system degradation.

The input particle flux to an MCP is typically inferred through the counting of individual charge clouds. Often, a charge-sensitive preamplifier followed by a discriminator is used to trigger an event counter when the total number of electrons in a charge cloud exceeds a pre-determined threshold [3,7]. Typically, individual charge clouds have time durations of $\sim 1-10 \mathrm{~ns}$, enabling $>100 \mathrm{MHz}$ counting with sufficiently fast electronics [19]. However, in particular for space-based applications, limited mass and power resources result in preamplifier/discriminator devices with $<50 \mathrm{MHz}$ counting $[7,20]$. Therefore, recovering the incident particle flux of high-intensity particle bunches becomes nontrivial.

In this article, we develop a parameterized model of MCP gain variation that enables the estimation of incident particle flux from the time-integration of the MCP output current. This technique eliminates the dead-time effects associated with the counting of individual pulses. We will demonstrate the effectiveness of this approach using laboratory measurements of an MCP's response to a pulsed charged particle beam. Although low energy electrons were used here as an incident particle source, the results of this study should be relevant for any MCP-based detection system, regardless of input particle species or photon wavelength.

\section{Model of Dynamic MCP Response}

In this section we provide an analytical model of the response of an MCP to large incident fluxes. We first demonstrate that charge-integration of the secondary electron current with respect to time can be used to estimate the incident flux with analogous statistical precision as a pulse counter. We then apply a simple model to describe the 89 evolution of the mean MCP gain in response to pulsed packets of incident particles. This 90 model will be used as a basis to interpret and scale laboratory measurements in section 3 . 


\subsection{Charge-Integration-Based Counting}

Pólya statistics have been used to model the distribution of secondary electrons produced from photomultiplier tubes and MCPs [21-23]. For non-zero incident particle flux, the analytical distribution reduces to a two-parameter Gamma distribution. We therefore describe the probability distribution function $(P)$ of the amount of charge $(q)$ in a secondary electron cloud as,

$$
P(q)=\frac{1}{\Gamma(\gamma) Q^{\gamma}} q^{\gamma-1} \exp -q / Q
$$

where $\Gamma$ is the Gamma function and the 'shape' and 'scale' of the distribution are defined by Gamma distribution parameters " $\gamma$ " and " $Q$ ", respectively. For the limits $\gamma \rightarrow 1$ and $\gamma \rightarrow \infty$, the distribution follows an exponential or Gaussian shape. We define the MCP gain as the mean value of this distribution, i.e., $G \equiv \gamma Q$. Any additional peaks in the distribution that are associated with electronics noise were not included in this description. We assume that the MCP voltage is sufficiently high that $G$ is much higher than any detection system noise [7].

Now consider $N$ particles that strike the MCP within time $\Delta t$. The total number of secondary electrons produced by the MCP will correspond to the sum of $N$ random samples from the distribution described by Eq. 1. These electrons are collected onto a conducting anode and form a measureable current (units $\mathrm{C} / \mathrm{s}$ ),

$$
I=q G \Phi_{i} A_{M C P} \varepsilon_{M C P},
$$

where $\Phi_{i}$ is the incident particle flux (units particles $/\left(\mathrm{cm}^{2} \mathrm{~s}\right)$ ), $A_{M C P}$ is the area of the $\mathrm{MCP}$ (units $\mathrm{cm}^{2}$ ), $q$ is the unit charge (units C), and $\varepsilon_{M C P}$ is the MCP efficiency (unitless).

The total number of electrons sampled over a finite time interval will also follow a Gamma distribution (Eq. 1). The mean $(\mu)$ and variance $\left(\sigma^{2}\right)$ of this distribution are $\gamma N Q$ (i.e., $N G$ ) and $\gamma N Q^{2}$, respectively $[24,25]$. The relative deviation of this distribution with respect to its mean is,

$$
\frac{\sigma}{\mu}=\frac{\sqrt{\gamma N Q^{2}}}{\gamma N Q}=\frac{1}{\sqrt{\gamma N}} .
$$

Following the central limit theorem, as $N$ increases, the total number of measured electrons approaches the mean value, i.e., $N G$. Typical MCP operation results in $\gamma \geq 1$ [23] such that this convergence will occur faster than it would for a Poisson distribution (i.e., $\left.\frac{1}{\sqrt{N}}\right)$. Therefore, instead of counting individual charge clouds via a discriminator circuit, the total number of particles that struck the MCP can also be estimated (with analogous statistical uncertainty) by integrating the total charge collected by the anode and dividing by the mean gain i.e., 


\subsection{MCP Gain Dependence on Incident Flux}

When an incident particle generates a secondary electron cascade inside an MCP channel, the charge must be replenished before that channel can discharge again. Due to the nature of the cascade, more charge tends to be depleted from the bottom of each channel. However, an overall replenishment time for a given detector configuration has provided reasonable description of MCP saturation [3]. The characteristic recovery time of each channel is often taken to be $\tau_{D} \approx R C$, where $R$ and $C$ are the resistance and capacitance of an MCP channel, respectively. For most MCPs, $\tau_{D}$ is on the order of a few milliseconds.

145 Channels in an MCP are semi-independent from one another such that if a given channel has been depleted of charge, its neighboring channels can still generate secondary electron cascades. Therefore, MCPs with large numbers of channels per $\mathrm{cm}^{2}$ are capable of counting at $\mathrm{MHz}$ rates rather than the $\mathrm{kHz}$ rates implied by $1 / \tau_{D}[3]$.

Consider a burst of incident flux $\Phi_{i}$ focused onto an area $A_{\mathrm{MCP}}$ of an MCP during an interval $\Delta t<<\tau_{D}$. We define the ratio of the number of incident particles to the number of MCP channels as,

Here, ' $d$ ' defines the center-to-center spacing of adjacent MCP channels and $\rho$ provides a unitless measure of the MCP's 'usage,' i.e., the ratio of channels in a given area expected to be at least partially discharged. The stacking of multiple MCP plates results in an effective coupling between neighboring channels as charge clouds between successive plates can spread into multiple channels. Because most charge is extracted lower down in the MCP stack, charge cloud spreading above the lowest plate can nonetheless result in substantial depletion of multiple channels simultaneously. If all channels were completely independent of one another, i.e., if a single plate were used, no degradation in a detection system's counting ability should be observed for $\rho<1$.

Following Eq. 2, the measured incident particle flux $\left(\Phi_{m}\right)$ is proportional to the total amount of charge collected by the anode divided by the accumulation time, area, and undistorted (i.e., low incident flux) average $\mathrm{MCP}$ gain $\left(\mathrm{G}_{\mathrm{o}}\right)$. To the degree that these assumptions are correct,

$$
\rho=\frac{\Phi_{i} A_{M C P} \Delta t}{\frac{A_{M C P}}{d^{2}}}=\Phi_{i} \Delta t d^{2} .
$$

$$
\Phi_{m}=\frac{\int_{0}^{\Delta t} I(t) d t}{A_{M C P} \Delta t G_{o}} .
$$

We define a critical number of particles per channel, $\rho_{o}$, where the MCP detection system performance has begun to degrade, i.e., the average gain has reduced to $50 \%$ of its nominal value. This parameter implicitly incorporates effects due to the spreading of the secondary electron charge cloud between successive MCP plates and the ratio of charge available for depletion to the average gain within a given channel. Such effects should remain constant for a given MCP stack geometry and operating voltage. The ratio of $\rho$ to 
$\rho_{o}$ is equivalent to the 'saturation parameter' as defined in the simple analytical model of MCP saturation by Giudicotti et al. [16]. Following the 'pulse mode' limit of their model, the variation of gain in terms of the undistorted gain and incident flux becomes,

Combining Eqs. 6 and 7 gives,

$$
\mathrm{G}=\frac{\mathrm{G}_{o}}{1+\frac{\rho}{\rho_{o}}}=\frac{\mathrm{G}_{o}}{1+\frac{\Phi_{i} \Delta t d^{2}}{\rho_{o}}}
$$

$$
\Phi_{m}=\varepsilon_{M C P} \frac{\Phi_{i}}{\Delta t} \int_{0}^{\Delta t} \frac{G(t)}{G_{o}} d t=\varepsilon_{M C P} \frac{\rho_{o}}{\Delta t d^{2}} \log \left(1+\frac{\Phi_{i} \Delta t d^{2}}{\rho_{o}}\right) .
$$

The incident flux can then be recovered from the measured flux using,

$$
\Phi_{i}=\frac{\rho_{o}}{\Delta t d^{2}}\left(\exp \left(\frac{\Phi_{m} \Delta t d^{2}}{\varepsilon_{M C P} \rho_{o}}\right)-1\right)
$$

In the limit of $\rho \ll \rho_{o}$, i.e., the low flux limit, equation (9) reduces to $\Phi_{i}=\Phi_{m}$. We expect that Eqs. 7-9 are valid up through $\rho / \rho_{o} \sim 1$. Higher incident fluxes (i.e., $\rho \gg \rho_{o}$ ) will result in channels being affected by more than one particle impact, leading to further distortion of the distribution of secondary electrons. The parameter $\rho_{o}$ is expected to be a function of MCP stack geometry and applied voltage (e.g., gain).

\section{Laboratory Results and Analysis}

Leveraging insights from the model developed in section 2, we analyzed laboratory measurements of an MCP stack using a pulsed electron beam. Tests were conducting at NASA Goddard Space Flight Center in the same facility that was used to calibrate the Dual Electron Spectrometer (DES) suite for the Fast Plasma Investigation (FPI) flying on NASA's Magnetospheric Multiscale (MMS) mission [7].

\subsection{Laboratory Setup}

The MCP stack used for testing, a flight spare from DES, consisted of two $1.5 \mathrm{~mm}$ thickness matched plates with $25 \mu \mathrm{m}$ diameter channels, $32 \mu \mathrm{m}$ center-to-center spacing (i.e., a channel density of $\sim 10^{5}$ channels per $\mathrm{cm}^{2}$ ), and a total stack resistance of $18 \mathrm{M} \Omega$. The front of the MCP stack was masked over, allowing only incident particles to reach the plates in a circular spot with $A \mathrm{MCP}=0.2 \mathrm{~cm}^{2}$, i.e., $\sim 20000$ channels. This stack was pre-conditioned through the extraction of $>1 \mathrm{C} / \mathrm{cm}^{2}$ such that its gain was expected to remain constant throughout testing $[7,26]$. A resistive divider provided appropriate biasing of the individual MCP plates using a high-voltage power supply.

A schematic of the laboratory test apparatus is shown in Figure 1. A $100 \mathrm{eV}$ electron beam was used to provide uniform particle flux over an area $>>A_{M C P}$. The electron flux was modulated using a set of parallel plates that, with sufficient voltage applied, deflected the beam away from the active area of the MCP. A voltage of $\sim 100 \mathrm{~V}$ (rising edge of $\sim 6 \mu \mathrm{s}$ ) was sufficient to completely redirect the incident electron beam. A Faraday cup mounted at a $45^{\circ}$ angle with respect to the MCP surface normal served as a 
beam monitor, providing absolute flux estimates. A rotation-stage motion system enabled alternating measurements between the Faraday cup and MCP.

A solid anode was incorporated into the MCP detector stack to collect the secondary electron current. The anode signal was passed through a high-voltage capacitor in order to re-reference the signal to ground, and then was routed, through a vacuum chamber feed through, to an inverting charge-sensitive preamplifier and finally captured with an oscilloscope. The oscilloscope acquisition signal was triggered from the rising edge of the beam modulator signal and was averaged over 512 pulses, providing a smoothed measurement of the anode current derived in equation (2).

\subsection{Dynamic Variation of MCP Gain}

To characterize the variation of MCP gain, the incident electron beam was pulsed with a spacing $(T)$ of $10 \mathrm{~ms}$. This spacing will be shown in section 3.4 to be well above the characteristic time for an MCP channel to replenish its charge. The beam flux was varied between $10^{6}$ and $10^{9} \mathrm{~cm}^{-2} \mathrm{~s}^{-1}$ by adjusting the voltage on the electron source from 1.2 to $1.7 \mathrm{~V}$. The relationship between the source voltage and Faraday-cup measured flux is shown in Figure 2a. At each flux setting, the corresponding inverted averaged anode current was captured with the oscilloscope for effective pulse durations of $4 \mu \mathrm{s}, 19 \mu \mathrm{s}$, and $44 \mu$ s (i.e., $10 \mu \mathrm{s}, 25 \mu \mathrm{s}$, and $50 \mu$ s set points with a $\sim 6 \mu$ s rise time). Anode currents only for the $44 \mu$ s pulses are shown in Figure $2 b$, as the smaller pulse times exhibited nearly identical peak shapes over their respective overlap with the longer pulse time. Consistent with previous studies [16-18], the measured anode current decreased with increased overall flux or increased time after the start of each pulse. This decrease was most notable for the highest flux settings.

The anode current shape at each flux setting was fit using a Levenberg-Marquardt nonlinear least squares algorithm using Eqs. 2 and 7. The free parameters were the incident flux, $\Phi_{\mathrm{i}}$, and the critical number of particles per channel, $\rho_{\mathrm{o}}$. The channel-channel spacing was taken as $d=32 \mu \mathrm{m}$ and the time since the start of the pulse was taken as $\Delta t$. The resulting fits are included in Figures $2 a$ and $2 b$. To avoid rise time edge effects and the deep saturation regime, the segments of each anode current curve used for fitting were limited to $\mathrm{t}>2 \mu \mathrm{s}$ and $\rho<2 \rho_{\mathrm{o}}$. For these data, the critical number of particles per channel was found to be $\rho_{0}=0.1$. Differences in the recovered flux levels are attributed to variation of the electron beam flux with time at a given voltage setting. The relative flux levels recovered from the model fits provide corrections for any variability of the beam between MCP and Faraday cup measurements.

Assuming that the dynamic reduction of MCP gain was only a function of the number of particles per channel, Eqs. 2 and 5 could be used to transform each $(t, I)$ value in Figure $2 \mathrm{~b}$ into $\left(\rho, G / G_{o}\right)$ space. These scalings, shown in Figure 3, indeed resulted in a single overall curve that described the variation of gain with incident particle flux. As predicted, the functional form of Eq. 7 provided a good approximation of this relationship up to $\rho \sim$ $3-5 \rho_{\mathrm{o}}$. Above this value, up to $\sim 10 \rho_{\mathrm{o}}$, the gain steepened with incident flux, reducing more quickly than the model. Near $\rho \sim 1$, the measured gain flattened, becoming larger than the modeled curve. Such variation suggested substantial distortion of the probability 
distribution function of gain at very high incident fluxes, and was consistent with the increased ratio of replenished charge to stored charge predicted by Giudicotti [18] for

\subsection{Integration-Based Counting}

Given the good agreement between laboratory measurements and the model developed in section 2, we could assess the viability of using Eqs. 8 and 9 to recover the incident flux from anode current. Here, we numerically integrated the total anode current measured at each flux setting for the $4 \mu \mathrm{s}, 19 \mu \mathrm{s}$, and $44 \mu \mathrm{s}$ pulses. In Figure $4 \mathrm{a}$, we compare these results with the values calculated from Eq. 8 using known incident fluxes, pulse durations, and critical numbers of particles per channel. The flux values used for this comparison were those estimated from the non-linear fitting of data in section 3.2.

The modeled curves accurately predicted the reduction of count rate due to reduced MCP gain and the corresponding improvement in performance when integrating pulses of a shorter duration, i.e., minimizing the total number of particles per channel. For the $4 \mu \mathrm{s}$ pulse, where even at high incident fluxes the value of $\rho$ remained less than unity (see Figure $4 \mathrm{~b}$ ), recoverable integration-based count rates up to $\sim 1 \mathrm{GHz}$ could be achieved.

\subsection{MCP Recovery Time}

Finally, to estimate the recovery time of an MCP channel $(\tau \mathrm{D})$, an incident flux of $\sim 10^{9}$ $\mathrm{cm}^{-2} \mathrm{~s}^{-1}$ was pulsed with an effective duration of $19 \mu \mathrm{s}(25 \mu \mathrm{s}$ beam chopper width with 6 $\mu$ s rise time), and the spacing ( $T$ ) of successive pulses was varied from 0.5 to $10 \mathrm{~ms}$. The average anode current for each spacing is shown in Figure 2a. As the spacing between pulses was reduced, the probability that particles would strike an already depleted channel increased, and the peak amplitude of the measured signal decreased. As shown in Figure $2 b$, the peak amplitude exhibited an $1-\exp \left(-\mathrm{T} / \tau_{\mathrm{D}}\right)$ dependence, enabling the estimation of $\tau_{\mathrm{D}}=1.7 \mathrm{~ms}$ from the measured data. This recovery time was consistent with an effective $100 \mathrm{pF}$ MCP capacitance given the stack resistance of $18 \mathrm{M} \Omega$.

\section{Discussion}

The critical number of particles per channel, $\rho_{0}$, parameterizes both channel-channel coupling effects and the number of particles that can initiate an electron cascade within a channel before fully depleting it. Consequently, adjustment of either the detection system geometry (e.g., channel density, plate-spacing, channel bias angle with respect to the MCP surface normal) or MCP operating point (e.g., gain per incident particle and electric field between stacked plates) may result in a change in $\rho_{0}$. Pre-conditioning of MCP plates via the extraction of $>1 \mathrm{C} / \mathrm{cm}^{2}$ is critical to ensure that the MCP gain (and corresponding parameter $\rho_{0}$ ) remains constant with time for a given operating setting $[7,26]$. Without this initial charge extraction, $\rho_{0}$ may vary with detection system lifetime.

As the electron charge cloud travels from the channel output to the anode, it generates an image current on the back surface of the MCP. This signal is equivalent to the anode current but with opposite polarity, enabling two independent measurements of incident particle flux. The image charge signal can provide a total MCP count rate in parallel with a segmented or delay-line anode system that provides additional information on the 
incident particle position [27]. In such a configuration, consider the case that a chargeintegrator is used to capture the total image current from a pulsed beam and traditional counters are used for the segmented or delay-line anode. For incident count rates well within the counter operating range and $\rho<<\rho_{0}$, both measurements can be compared to provide a relative calibration between them. When the incident count rate increases beyond the capabilities of the preamplifier/discriminators (i.e., > $50 \mathrm{MHz}$ ), the chargeintegrator measurement will continue to provide reliable estimates of the total incident flux, extending the dynamic range of the detection system.

The charge-integrator acts as a non-paralyzable counter, providing a key advantage over conventional pulse counting techniques. Consider the 20,000 channels in the presented experiment. Because each channel is semi-independent, they can all be discharged simultaneously. Integration-based counting is therefore only limited by the ability to pulse the incident beam. Although the electron beam modulation here was limited to $\sim 4$ $\mu$ s, enabling stable operation for $\sim 1 \mathrm{GHz}$, nanosecond-level particle gating could enable effective counting up to $\sim 1 \mathrm{THz}$, where the MCP output resembles a constant current level rather than a series of individual pulses. In this mode of operation, the MCP acts as a charge-amplifying Faraday cup. Provided that the integration window is larger than that of the incident flux, there should be no degradation in counting from the finite pulse width of the secondary electron cloud.

Finally, although the experimental results here utilized energy electrons as the source of incident flux, the variation of MCP gain should be somewhat species and/or photon wavelength independent. Because the charge cloud generated by an incident particle is comprised of MCP channel electrons, the physics of the secondary electron cascade remains unchanged, and similar MCP operating gains of $\sim 10^{6}$ have been achieved for UV photons, electrons, ions, and energetic particles [3]. Furthermore, the anode currents presented here are similar in structure to those reported by Coeck et al. [17], who utilized high-intensity ion bunches rather than low energy electrons. We note that low energy sensors are more likely to immediately benefit from this technique, as it is more straightforward to modulate the incident particle flux.

\section{Conclusions}

We have developed a parameterized model of MCP gain that describes its variation in terms of the number of incident particles per channel within a detector recovery time. This model has been validated using laboratory measurements of an MCP's response to a pulsed electron beam, but should be applicable to any MCP-based detection system. Integration of the MCP anode current under these conditions has been demonstrated to provide recoverable count rates up to $\sim 1 \mathrm{GHz}$, providing more than an order of magnitude improvement over typical space-based counting electronics. This technique leverages pulsed $\mathrm{MCP}$ operation to significantly extend the dynamic range of low energy MCP-based sensors.

Acknowledgements. This work was supported by the Internal Research and Development Program at NASA Goddard Space Flight Center. The authors would like to acknowledge B. L. Giles and T. E. Moore for their administrative support of this project. 
1. Wüest, M., Evans, D. S., and von Steiger, R.,ESA Publications Division, (2007).

2. Goodrich, G. W. and Wiley, W.C. Rev. Sci. Instrum. 33, 761 (1962).

3. Wiza, L. J., Nucl. Instrum. Meth., 162, 587 (1979).

4. Siegmund, O.H.W., Coburn, K., and Maline, R. F., IEEE Trans. Nucl. Sci., 32, 443 (1985).

5. Fraser, G. W., Pearson, J. F., and Lees, J.E., IEEE Trans. Nucl. Sci., 35, 529 (1988).

6. Eberhardt, E.H., App. Opt., 18, 1418 (1979).

7. Pollock, C. J. et al., Space. Sci. Rev., 199, 331 (2016).

8. Meier, R. and Eberhardt, P., Int. J. Mass. Spec. Ion. Proc., 123, 19 (1993).

9. Oberheide, J., Willhelms, P., and Zimmer, M., Meas. Sci. Technol. 8, 351 (1997).

10. Yagi, S., Nagata, T., Koide M., Itoh, Y., Koizumi, T., and Azuma, Y., Nucl. Instrum. Meth. Phys. Res., 183, 476 (2001).

11. Fraser, G. W., Int. J. Mass. Spec., 215, 13 (2002).

12. Fraser, G. W., Pain, M. T., and Lees, J. E., Nucl. Instrum. Meth. Phys. Res., 327, 328 (1993).

13. Fraser, G. W., Pain, M. T., Lees, J. E., and Pearson, J. F., Nucl. Instrum. Meth. Phys. Res., 306, 247 (1991).

14. Tremsin, A. S., Pearson, J. F., Fraser, G. W., Feller, W. B., and White, P., Nucl. Instrum. Meth. Phys. Res., 379, 139 (1996).

15. Giudicotti, L. Nucl. Instrum. Meth. Phys. Res., 480, 670 (2002).

16. Giudicotti, L. Rev. Sci. Instrum., 65, 247 (1994).

17. Coeck, S., Beck, M., Delauré, B., Golovko, V.V., Herbane, M., Lindroth, A., Kopecky, S., Kozlov, V.Y., Kraev, I.S., Phalet, T., and Severijns, N., Nucl. Instrum. Meth. Phys. Res., 557, 516 (2006).

18. Giudicotti, L. Nucl. Instrum. Meth. Phys. Res., 659, 336 (2011).

19. Cova, S., Ghioni, M., and Zappa, F., Rev. Sci. Instrum., 62, 2596 (1991).

20. McFadden, J. P., Carlson, C. W., Larson, D., Ludlam, M., Abiad, R., Elliott, B., Turin, P., Marckwordt, M., and Angelopoulos, V., Space Sci. Rev., 141, 477 (2009).

21. Stokey, R. J., and Lee, P.J., TDA Progress Report, 42 (1983)

22. Anderson, W.S., Armitage, J.C., Chevreau, P., Heinrich, J.G., Lu, C., McDonald, I., McDonald, K.T., Miller, B., Secrest, D., and Weckel, J., Proc. Symposium on Detector Research and Development for the Superconducting Super Collider, 222 (1991).

23. Gershman, D. J., Gliese, U., Dorelli, J. C., Avanov, L.A., Barrie, A.C., Chornay, D.J., MacDonald, E. A., Holland, M. P., Giles, B.L., and Pollock, C.J., J. Geophys. Res.: Space Phys., 121, 10,005 (2016).

24. Stacy, E.W., Ann. Math. Stat., 33, 1187 (196).

25. Gupta, R.D., and Kundu, D., Australian \& New Zealand Journal of Statistics, 41, 173 (1999).

26. Mackler, D.A., Avanov, L.A., Barrie, A.C., Chornay, D.J., Gershman, D.J., Giles, B.L., Pollock, C.J., Rager, A., and Smith, S., Planet. Space Sci. (in revision) 
27. Jagutzki, O., Mergel, V., Ullmann-Pfleger, K., Spielberger, L., Meyer, U., Doerner, R., and Schmidt-Boecking, W., Proc. SPIE 3438, 322 (1998).

\section{Figures and Captions}

Figure 1. Schematic of experimental setup used for MCP testing. Secondary electrons from an MCP stack illuminated with a pulsed electron beam were collected onto a solid anode. The resultant anode current was captured with an oscilloscope and compared with model predictions. A Faraday cup beam monitor (not shown) was mounted $45^{\circ}$ from the MCP surface normal direction. A motion-control mechanism was used to alternate between the beam monitor and MCP measurements.

Figure 2. (a) Incident particle flux as a function of voltage applied to the electron beam source. The black and red curves correspond to measurements from the Faraday cup beam monitor and values derived from fits to the anode current, respectively. (b) Average inverted anode current as a function of time from the start of a $44 \mu$ s incident electron pulse. Different colors represent different incident electron fluxes. Best-fit modeled curves for each flux setting are shown as blacked dashed lines in the sub-panel of curves in log-current space. The good agreement between the modeled and measured curves indicates that our parameterization of MCP gain is appropriate.

Figure 3. Relative $\mathrm{MCP}$ gain (i.e., $\mathrm{G} / \mathrm{G}_{0}$ ) as a function of incident particles per channel, $\rho$. The anode current curves from Figure $2 b$ were transformed from $(t, I)$ to $\left(\rho, G / G_{0}\right)$-space using the analytical model derived in section 2 . A self-similar shape across all incident flux settings validates our parameterization of gain as primarily a function of $\rho$.

Figure 4. (a) Effective count rate derived through numerical integration of the anode current for $44 \mu \mathrm{s}, 19 \mu \mathrm{s}$, and $4 \mu \mathrm{s}$ pulses as a function of incident flux. The measured data (solid dots) are shown with corresponding modeled curves (dashed lines). At the highestflux setting of $3.3 \times 10^{9} \mathrm{~cm}^{-2} \mathrm{~s}^{-1}$ (vertical dotted line), the time series of anode current is shown in (b). All pulses exhibit the same fundamental anode current shape but with a higher average value for the shorter pulses due to their reduced number of particles per channel. With a $4 \mu \mathrm{s}$, effective count rates up to $\sim 1 \mathrm{GHz}$ could be achieved.

Figure 5. (a) Anode current as a function of time for pulse spacings ( $\mathrm{T}$ ) between $0.5 \mathrm{~ms}$ and 10ms. (b) The relative amplitude of the anode current as a function of T. A model fit of the form 1-exp(-T/ $\left.\tau_{\mathrm{D}}\right)$ is shown with a black dashed line, indicating an effective MCP channel recovery time of $\tau_{\mathrm{D}}=1.7 \mathrm{~ms}$. 


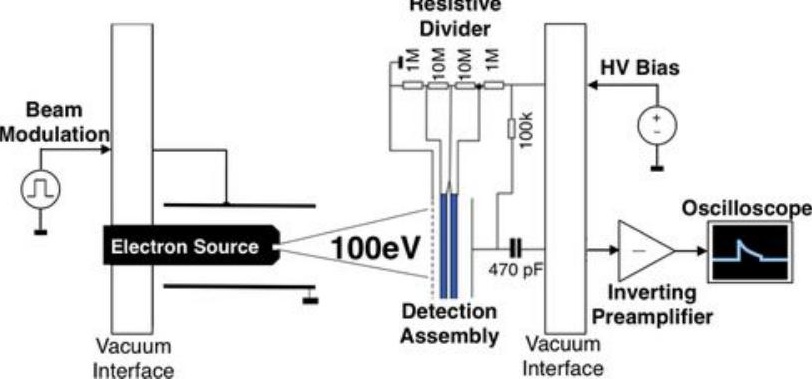




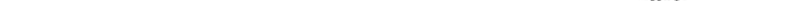




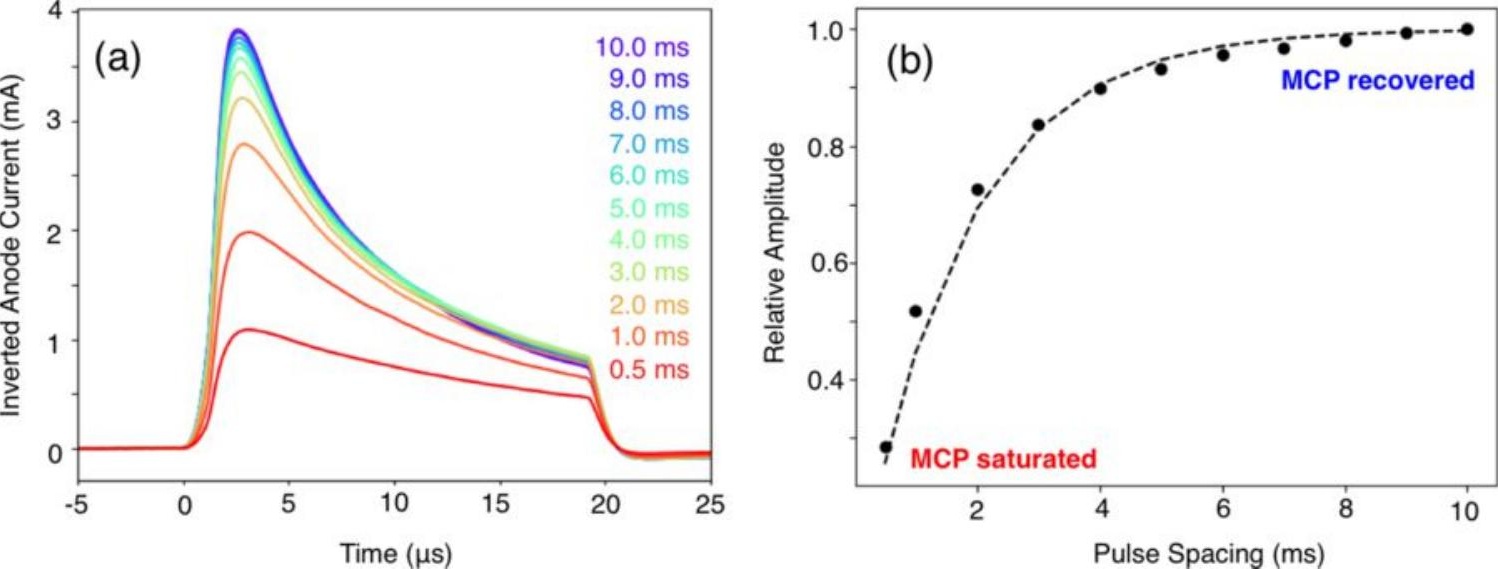

